HNO 2010 • 58:198-199

DOI 10.1007/s00106-009-2073-2

Online publiziert: 5. März 2010

(c) Springer-Verlag 2010

\author{
H. Maier \\ Bundeswehrkrankenhaus UIm
}

\section{Sialadenitis und Sialolithiasis}

Entzündungen der großen Kopfspeicheldrüsen sind insgesamt betrachtet nicht selten, können differenzialdiagnostisch und/oder therapeutisch aber bisweilen Probleme bereiten. Sie können durch bakterielle und virale Infektionen, aber auch durch Autoimmunprozesse ausgelöst werden. Mit Problemkeimen muss vermehrt gerechnet werden.

\section{( ) Mit Problemkeimen muss vermehrt gerechnet werden}

Unter den bakteriellen Sialadenitiden bereitet die Diagnostik der akuten eitrigen Sialadenitis aufgrund des eindeutigen klinischen Bildes meist keine Schwierigkeiten. Entscheidend ist, dass einerseits eine relevante Obstruktion des Gangsystems z. B. durch Stenosen oder Steine und andererseits Abszesse erkannt und beseitigt bzw. drainiert werden. Dies kann heute oft durch minimal-invasive sialendoskopische Techniken erfolgen [3]. Darüber hinaus müssen eine ausreichende Flüssigkeitszufuhr, eine Stimulation der Speichelsekretion und eine sofortige Antibiotikatherapie erfolgen. Meist handelt es sich um eine aszendierende Infektion mit Staphylococcus aureus. Daher sollte initial grundsätzlich ein staphylokokkenwirksames Antibiotikum eingesetzt werden. Eine mikrobiologische Untersuchung ist jedoch unerlässlich, da zahlreiche andere, z. T. auch multiresistente Keime beteiligt sein können [1].

Wesentlich problematischer sind mykobakterielle Infektionen, mit denen heute infolge eines vermehrten Tourismus in Länder der 3. Welt und einer verstärkten Zuwanderung von Emigranten aus Afrika, Asien und Osteuropa wieder ge- rechnet werden muss. Die Speicheldrüsentuberkulose ist häufig symptomarm und imponiert durch eine langsame tumoröse Schwellung, meist der Gl. parotis. Entsprechend erfolgt aufgrund der Verdachtsdiagnose Speicheldrüsentumor nicht selten ein unnötiger operativer Eingriff. Letzteres kann durch den gezielten kombinierten Einsatz von FNAC und PCR vermieden werden. Diese Kombination weist eine hohe Spezifität und Sensitivität bei diesen Infektionen auf [4].

In früheren Jahren war der Begriff virale Sialadenitis nahezu synonym mit einer Infektion mit dem Mumpsvirus. Infektionen mit anderen sialotropen Viren waren die absolute Ausnahme. Diese Situation hat sich mittlerweile wesentlich verändert. Die virale Sialadenitis hat durch die Zunahme der HIV- und Hepatitis-CInfektionen in den letzten Jahrzehnten einen Gestaltwandel erfahren. Eine chronische Infektion mit dem Hepatitis-CVirus führt in vielen Fällen zu einer klinisch symptomarm verlaufenden Begleitsialadenitis, die nicht selten mit einer erheblichen Reduktion der Speichelsekretion und konsekutiver Mundtrockenheit einhergeht. Dies trifft auch für die HIVInfektion zu. Neben der Mundtrockenheit kann diese Form der Virussialadenitis ausgeprägte morphologische Veränderungen in der Gl. parotis verursachen. Hier sind v. a. multizentrische Parotiszysten, die zystische lymphoide Hyperplasie, die benigne lymphoepitheliale Läsion, die Lymphadenopathie der Parotis und die myoepitheliale Sialadenitis zu nennen (Übersicht bei [5]). Vor allem wenn zystische Veränderungen oder multiple Lymphknotenschwellungen in der Gl. parotis durch bildgebende Untersu- chungen festgestellt werden, muss heutzutage eine HIV-Infektion ausgeschlossen werden.

\section{( Die virale Sialadenitis hat einen Gestaltwandel erfahren}

Im Hinblick auf die Therapie der Virussialadenitis haben sich in den letzten Jahren keine entscheidenden neuen Ansätze ergeben. Wenn möglich, wie z. B. bei der HIV- oder Hepatitis-C-Infektion, erfolgt eine medikamentöse Therapie der Grund-erkrankung. Die Therapie der Virussialadenitis selbst ist nach wie vor in erster Linie symptomatisch. Eine chirurgische Therapie bei HIV-assoziierten Speicheldrüsenerkrankungen wie z. B. Parotiszysten sollte nur ausnahmsweise und v. a. dann erfolgen, wenn zusätzlich der Verdacht auf eine Tumorerkrankung besteht.

Eine chronische Speicheldrüsenentzündung, die möglicherweise im Gefolge einer Virusinfektion auftritt, stellt die Autoimmunsialadenitis dar. Sie manifestiert sich meist im Rahmen des SjögrenSyndroms und ist mit einer Prävalenz von 0,5-1,0\% eine häufige Erkrankung, die meist erst spät diagnostiziert wird und $\mathrm{zu}$ einer erheblichen Einschränkung der Lebensqualität führen kann [6]. Der Nachweis von Autoantikörpern im Serum, einer eingeschränkten Sekretionsleistung von Speichel- und Tränendrüsen und typischen pathomorphologischen Veränderungen im Speicheldrüsengewebe sind für die Diagnosestellung richtungweisend. Charakteristische Veränderungen des Speicheldrüsengangsystems lassen sich heute am besten mit der MRT-Sialographie darstellen. Die Sonographie zeigt 
typischerweise eine heterogene Echogenität des Drüsengewebes und etabliert sich zunehmend in der Diagnostik der Autoimmunsialadenitis. Dieses Verfahren ist auch für das Follow-up der Patienten mit Autoimmunsialadenitis, die ein erhöhtes Risiko für eine intraglanduläre Manifestation eines Non-Hodgkin-Lymphoms aufweisen, hervorragend geeignet.

Die Therapiemöglichkeiten bei der Autoimmunsialadenitis sind bislang beschränkt und überwiegend symptomatisch ausgerichtet. Eine systemische Therapie mit sog. krankheitsmodifizierenden antirheumatischen Medikamenten, die beim Vorliegen extraglandulärer Manifestationen eingesetzt werden, hat keine positive Wirkung auf die eingeschränkte Speichelsekretion. Einen Hoffnungsschimmer liefert möglicherweise der therapeutische Einsatz von monoklonalen Antikörpern. In ersten Therapiestudien mit Rituximab und Epratuzumab konnte eine klinische Besserung der Sicca-Symptomatik erreicht werden $[7,8]$.

\section{$>$ Die Therapie der Auto- immunsialadenitis ist überwiegend symptomatisch}

Eine weitere Form der chronischen Sialadenitis stellt die chronisch rezidivierende Parotitis dar, die in einer juvenilen und adulten Form auftritt. Nach wie vor ist die Ätiologie dieser Erkrankung weitgehend unklar. Diskutiert werden neben genetischen Faktoren und Fehlbildungen chronische bakterielle Infektionen mit einer persistierenden subklinischen Entzündungsreaktion. Die Diagnose wird bis heute weitgehend anhand von Anamnese und klinischem Befund gestellt. Unter den bildgebenden Verfahren stellt die hochauflösende Sonographie das Verfahren der Wahl dar. Mit der Einführung der Sialendoskopie wurde in den letzten Jahren eine neue Untersuchungstechnik etabliert, mit deren Hilfe die für das Krankheitsbild typischen Strikturen und Stenosen des Gangsystems dargestellt werden können [3]. Darüber hinaus ermöglicht dieses Verfahren eine gezielte Dilatation von stenotischen Gangabschnitten, eine Spülung des Gangsystems und die Instillation von Kortisonlösungen. Mit dieser Vorgehensweise, in Kombination mit einer Anregung der Speichelsekretion und einer gezielten Antibiotikatherapie, lässt sich in den meisten Fällen heute eine subtotale Parotidektomie vermeiden.

Die Sialendoskopie stellt jedoch nicht nur einen entscheidenden Fortschritt in der Diagnostik und Therapie von chronisch obstruktiven entzündlichen Speicheldrüsenerkrankungen, sondern auch für sonstige obstruktive Speicheldrüsenerkrankungen einschließlich der Sialolithiasis dar. Bei diesen Krankheitsbildern kann nach derzeitiger Einschätzung die interventionelle Sialendoskopie, insbesondere in Kombination mit anderen Therapiemodalitäten, einen Therapieerfolg in über 90\% der Fälle bei Erhalt der betroffenen Drüse ermöglichen $[2,3]$.

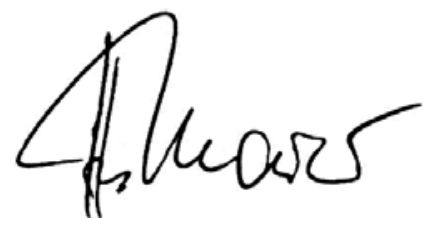

H. Maier

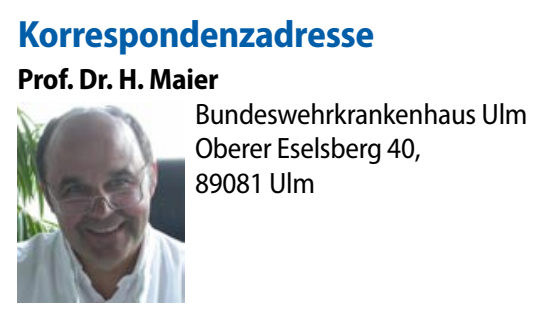

\section{Literatur}

1. Brook I (2009) The bacteriology of salivary gland infections. Oral Maxillofac Surg Clin North Am 21:269-274

2. Iro H, Zenk J, Escudier MP, Nahlieli O, Capaccio P, Katz P, McGurk M (2009) Outcome of minimally invasive management of salivary calculi in 4691 patients. Laryngoscope 119:263-268

3. Koch M, Iro H, Zenk J (2009) Sialendoscopy-based diagnosis and classification of parotid duct stenoses. Laryngoscope 119:1696-1703

4. Kim YH, Jeong W-J, Jung K-Y et al (2005) Diagnosis of major salivary gland tuberculosis: Experience of eight cases and review of the literature. Acta Otolaryngol 125:1318-1322

5. Maier H (2001) Therapie nichttumoröser Speicheldrüsenerkrankungen. Verhandlungsbericht 2001 Deutsche Gesellschaft für Hals-Nasen-Ohren-Heilkunde, Kopf- und Hals-Chirurgie. Koch U (Hrsg) Laryngorhinootologie 80(Suppl):89-114
6. Meijer JM, Meiners PM, Huddlestone Slater JJ et al (2009) Health related quality of life, employment and disability in patients with Sjögren's syndrome. Rheumatology 48:1077-1082

7. Meijer JM, Pijpe J, Bootsma H, Vissink A, Kallenberg CG (2007) The future of biologic agents in the treatment of Sjögren's syndrome. Clin Rev Allerg Immunol 32:292-297

8. Steinfeld SD, Tant L, Burmester GR et al ( 2006) Epratuzumab (humanised anti-CD22 antibody) in primary Sjögren,s syndrome: an open-label phase I/II study. Arthritis Res Ther 8:R 129 